\title{
Network Density based Analysis of MANET Routing Protocols
}

\author{
Rupali Gupta \\ Research Scholar \\ University college of Engineering, \\ Punjabi University, Patiala
}

\author{
Himanshu Aggarwal, Ph.D. \\ Professor \\ University college of Engineering, \\ Punjabi University, Patiala
}

\begin{abstract}
Mobile Ad-hoc Network (MANET) is a network with structure less self organizing an autonomous system of mobile nodes which are connected by wireless links. In this work an attempt has been made to compare the performance of three MANET Routing Protocols, such that Pro-active Routing Protocol: OLSR (Optimized Link State Routing Protocol), Reactive Routing Protocol: AODV (Ad-Hoc on Demand Distance Vector), Hybrid Routing Protocol: GRP (Geographic Routing Protocol) by using two different applications i.e. High Definition Video Conferencing and High Load FTP generating different types of data in the networks under different nodes densities $(20,40,60$ and 80$)$ in the networks. All the networks are simulated by using a discrete event simulator OPNET 14.0 and results are gathered by using different performance evaluation metrics. After the intensive simulation, it has found that the hybrid protocols (GRP) outperforms both reactive (AODV) and proactive (OLSR) protocols.
\end{abstract}

\section{General Terms}

Network density based analysis by using different MANET Routing Protocols

\section{Keywords}

MANET, OLSR, AODV, GRP, OPNET

\section{INTRODUCTION}

A network is an assemblage of people or systems or organizations who considered together as being related in some way who tend to contribute their information collectively for their business purpose which can be done as wired or wireless. Ad-hoc networks are wireless networks where nodes can share their information with each other. Adhoc networks form spontaneously without a need of an underlying structure or centered controller. A routing protocol is a protocol that specifies how routers communicate with each other, disseminating information that enables them to select routes between any two nodes on a computer network. Each router has a priori knowledge only of networks attached to it directly. A routing protocol shares this information first among immediate neighbours, and then throughout the network. This way, routers gain knowledge of the topology of the network. An ad hoc routing protocol is a convention, or standard, that controls how nodes decide which way to route packets between computing devices in a network [24].

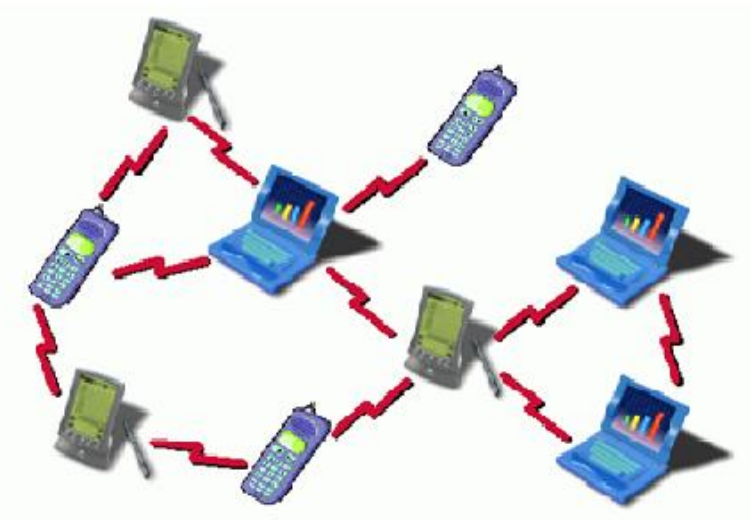

Fig.1. Layout of a MANET Network

A Mobile Ad-hoc network (MANET) is a multi hop wireless network designed by a group of mobile node that have Wireless features. MANET is an assemblage of wireless nodes that dynamically create a wireless network among them without any infrastructure. Ad-hoc is an imparted mode that allows computers to directly interchanged information with each other without a router. In Latin, ad-hoc means "for this" meaning "for this special purpose". In ad hoc networks, nodes do not start out familiar with the topology of their networks; instead, they have to discover it [13]. Mobile ad-hoc network also called a Mobile Mesh Network. It is a self configuring N/W of mobile devices connected by wireless links.

\subsection{Objectives}

Objectives of the dissertation are:

1. To study the working of various existing routing protocols for MANETs.

2. To analyze various MANET networks with varying nodes densities and configuration of the networks will be done by using MANET routing protocols.

3. Various performance evaluation metrics will be chosen to evaluate the networks.

\section{LITERATURE SURVEY MANET}

MANET (Mobile Ad Hoc Network) is a type of Ad Hoc network. The main issue of MANET is the breakage of link at certain moment and re-generation of link at certain state as it consists of routers, which are mobile in nature that are independent to roam in an arbitrary motion. 
1. On MANET Routing Protocols for Mobility and Scalability by Ashish Shrestha and Firat Tekiner [2]. They have concluded that with regards to overall performance, AODV and OLSR performed pretty well. However, AODV showed better efficiency to deal with high congestion and it scaled better by successfully delivering packets over heavily trafficked network compared to OLSR and TORA.

2. The efficiency evaluation and comparison of AODV, DSDV are carried out by Qingting Wei and Hong Zou [19]. It has been concluded that the increasing number of nodes, terrain size and maximum node speed will all bring the decrease in the efficiency of routing protocols. It has been analyzed that performance of AODV remained only $25 \%$ of DSDV when the number of nodes is varying and about $60 \%$ of DSDV when the maximum node speed is varying.

3. Performance Analysis of Routing Protocols for Wireless Ad-Hoc Networks was carried out by Sukhchandan Lally and Ljiljana Trajkovic [25]. On the basis of simulation result it have been conclude that AODV is the most flexible protocol among the three routing protocols and performs better in presence of movement while generating low routing traffic overhead. Scaling of MANET routing protocols such as AODV, DSR, and OLSR depends on node count, node density, traffic intensity, traffic path hop count, and network bandwidth.

\subsection{Types of MANET Routing Protocols}

MANET routing protocols are classified into following categories shown in fig 2 .

Classification of MANET routing protocols is:

1. Reactive Routing Protocols/On-demand

2. Pro-active routing protocol/ Table-driven

3. Hybrid routing protocol

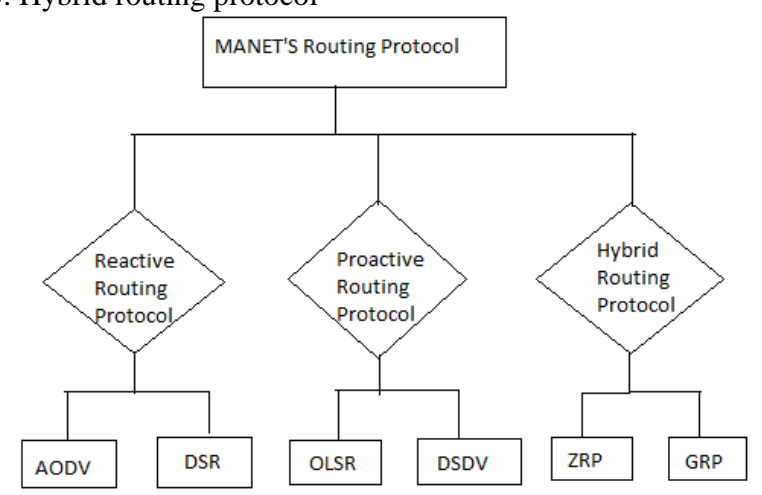

Figure 2. MANET Routing Categories and Protocols

\section{DESCRIPTION OF ROUTING PROTOCOLS}

\subsection{Reactive Routing Protocols}

Re-active routing protocols are source-initiated or on demand routing protocol. It means that every time a message is sent it first has to discover a way by searching the entire network. These routing protocols were accomplished to diminish the stress in proactive protocols by maintaining information for active routes only. Route discovery usually occurs by flooding a route request packets through the network. When a node with a route to the destination (or the destination itself) is reached a route reply is sent back to the source node using link reversal if the route request has traveled through bidirectional links or by piggy-backing the route in a route reply packet via flooding [13]. Main Appearance of this protocol is: finding short path, low-overhead communication, and load-balancing. Reactive routing protocols have further different categories.

\subsubsection{AODV}

It is a reactive on-demand routing protocol. This protocol works as on-demand mechanism for route discovery and route maintenance.

In route discovery mechanism involves route request (RREQ) and Route Reply (RREP) packets. Size of data packet being sent is of few bytes. In this protocol there is no route cache to maintain.

The following fields exist in each route table entry of AODV [5]:

- Destination IP Address: The IP address of the destination for which a route is supplied

- Destination Sequence Number: It is associated to the route.

- Next Hop: Either the destination itself or an intermediate node designated to forward packets to the destination

- Hop Count: The number of hops from the Originator IP Address to the Destination IP Address

- Lifetime: The time in milliseconds for which nodes receiving the RREP consider the route to be valid

- Routing Flags: The state of the route; up (valid), down (not valid) or in repair.

\subsection{Proactive Routing Protocol}

Proactive routing protocol is table driven routing protocol. This protocol maintains up to date information of all the routes in the network in a routing table. In table driven protocol requires each node to maintain one or more tables to store the information. Proactive routing protocols are not suited for large networks. Proactive routing protocol is further classified into different categories that are: OLSR, DSDV etc.

\subsubsection{OLSR (Optimized Link State Routing Protocol)}

OLSR comes under the category of Proactive Routing Protocol. It is a table driven routing protocol. In this protocol information of all the routes in the network is stored in a route table. This protocol works on the basis of link state algorithm. In pure link state protocol, all the links with neighbor nodes are asserted and are immersed in the whole network. The periodic nature of the protocol produces a large amount of overhead. In order to diminish the overhead, it limits the number of mobile nodes that can forward network wide traffic and for this purpose it uses Multi Point Relays (MPRs), which are reliable for forwarding routing messages. Mobile nodes which are selected as MPRs, can forward control traffic and diminish the size of control message. OLSR has three types of control messages that are: Hello Message: This control message is transmitted for sensing the neighbor and for Multi Point Distribution Relays (MPR) calculation.0

Topology Control Message (TC): Topology Control (TC) Message contains the MPR selector set information of a particular node. These TC messages are broadcast periodically within the TC interval, to other MPRs, which can further relay the information to their MPRs. Multiple Interface Declaration (MID), MID message contains the list of all IP addresses, used by any node in the network. All the nodes running OLSR transmit these messages on more than one interface. 


\subsection{Hybrid protocol}

Hybrid protocols exploit the strengths of both reactive and proactive protocols and combine them together to get better results. Different kind of protocols comes under this category are: ZRP, GRP.

\subsubsection{GRP (Gathering routing protocol)}

GRP is a type of position based protocol. It belongs to proactive routing protocol. Each position of the node will be noticeable by GPS and flooding will be optimized by quadrants. Flooding position updates on distance the node moved and neighborhood crossings. A hello protocol will be exchanged between nodes to identify their neighbors and their positions. At the same time, by means of route locking a node can return its packet to the last node when it can't keep on sending the packet to the next node [11].

\section{METHODOLOGY}

1. To meet the objectives, a structured methodology has followed. A thorough literature has done to gather the information about the MANETs and about the challenges in MANETs.

2. After defining the problem about the evaluation of routing protocols under different node densities, a deep analysis has done to get the optimized number of nodes and optimized configuration parameters for chosen protocols that could be simulated according to the available hardware resources.

3. After gathering the data about the configuration of simulation parameters various network models were designed with varying node densities $(20,40,60$, and 80$)$ by using a discrete event simulator 'OPNET'.

3. All the designed networks were configured with chosen protocols, such that AODV, OLSR and GRP with chosen optimized configuration parameters.

4. To evaluate the performance and to gather the results of the designed networks, various performance evaluation metrics, such as load and throughput have chosen.

5. After gathering the results of all the performance evaluation metrics in graphical and tabular form, conclusion has drawn and future scope about the improvement of the given work has proposed.

\section{NETWORK DESIGN AND PERFORMANCE MATRICES}

Following Performance matrices are used for the comparison of MANET routing protocols

Load: Represents the total load (in bits/sec) submitted to wireless LAN layers by all higher layers in all WLAN nodes of the network.

Throughput: Throughput is defined as; the ratio of the total data reaches a receiver from the sender. The time it takes by the receiver to receive the last message is called as throughput. Throughput is expressed as bytes or bits per sec (byte/sec or bit/sec). Some factors affect the throughput as; if there are many topology changes in the network, unreliable communication between nodes, limited bandwidth available and limited energy [27]. A high throughput is absolute choice in every network.

\section{SIMULATION MODEL (ENVIRONMENT) AND PARAMETERS}

The simulation is focused on the performance of MANET routing protocols, when node density is changed (20,40,60, and 80 ). For the simulation 1000x1000 meter campus network has used in which nodes are randomly placed within an environment with a fixed WLAN application server. Numbers of nodes are configured by using reactive protocol (AODV), proactive protocol (OLSR), and hybrid protocol (GRP) by setting different parameters provided by OPNET. To generate data in the network video conferencing and high load FTP applications are configured by using application node provided by MANET module in the OPNET simulator. To support the application, two fixed servers are configured and all the nodes in the network are configured according to the servers. Profiles for the configured application are defined by using profile definition node. The trajectory that is used to define the movements of the nodes is vector. Vector trajectory is used to simulate the real life behavior of the movements of the nodes. Various performance evaluations have chosen to gather the results in graphical form.

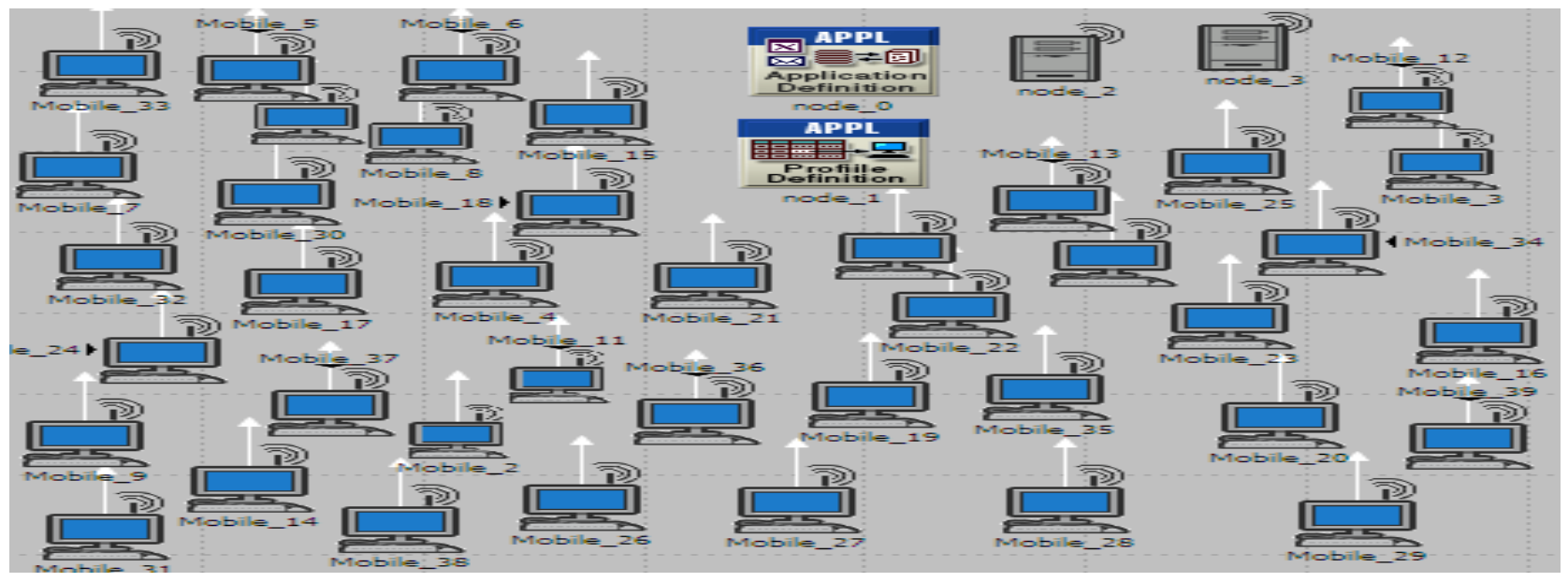

Figure 3: network model design using 40 nodes 


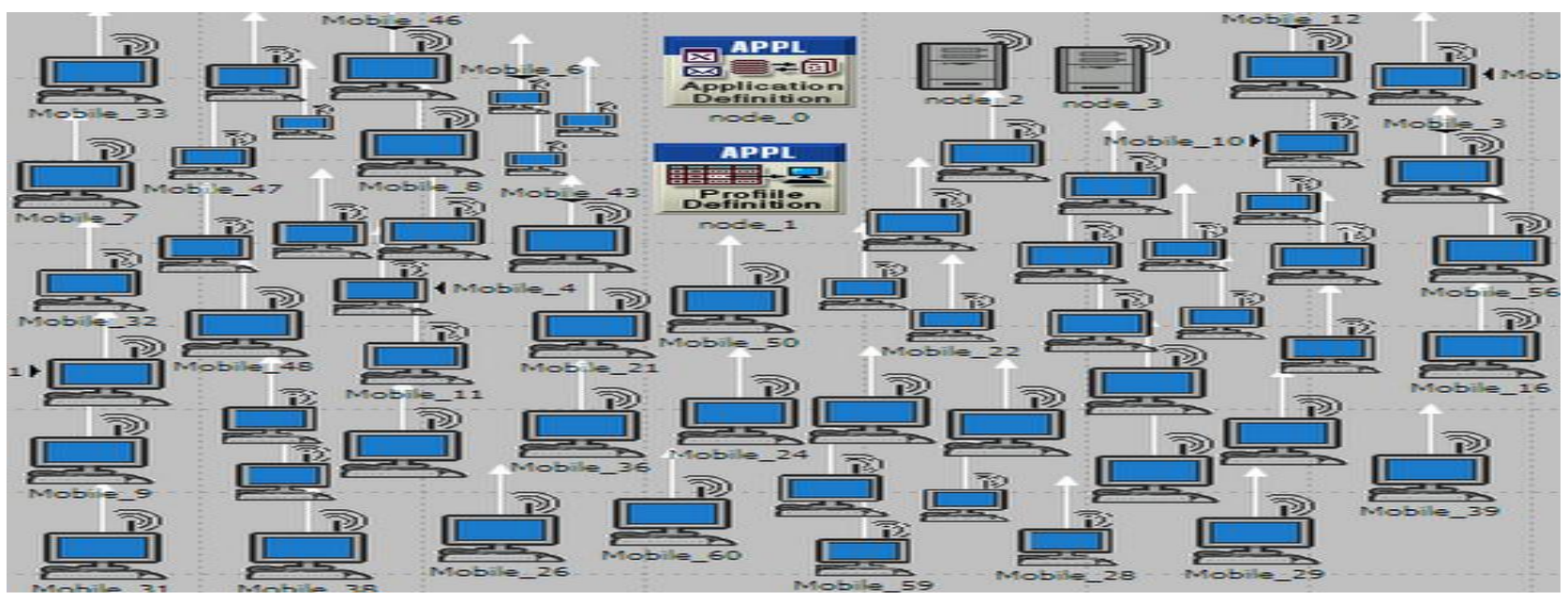

Figure 4: Network model design with 60 nodes

\subsection{Simulation Parameters:}

\begin{tabular}{|c|c|}
\multicolumn{2}{|c|}{ Table 1: simulation parameters } \\
\hline Attribute & Value \\
\hline $\begin{array}{c}\text { Maximum Simulation Time } \\
(\mathrm{sec})\end{array}$ & 300 \\
\hline Network Area & $1000 * 1000$ meters \\
\hline Mobility Model & Random Way Point \\
\hline No. of Nodes & GRP, AODV, OLSO 00 \\
\hline Protocols & High Load FTP, VIDEO \\
CONFERENCING
\end{tabular}

\section{SIMULATION RESULTS AND}

\section{ANALYSIS}

Simulation based analysis of different MANET routing protocols with varying number of nodes in a network environment. Used number of nodes is: $20,40,60,80$.
7.1. Result of AODV Protocol: The Analysis of AODV routing protocol have done with varying $(20,40,60,80)$ number of nodes. AODV protocol was simulated in all the four scenarios by using two parameters such as: Load, Throughput. These two parameters can be checked in all the 20, 40, 60, 80 mobile nodes.

Load: In fig. 5 It is concluded that network with 40 mobile nodes shows more load than 20, 60, 80 mobile nodes.

Throughput: In fig. 6 it is concluded that throughput for 20 mobile nodes in network has more throughput than $40,60,80$ Mobile nodes. AODV performs better with lesser network density.

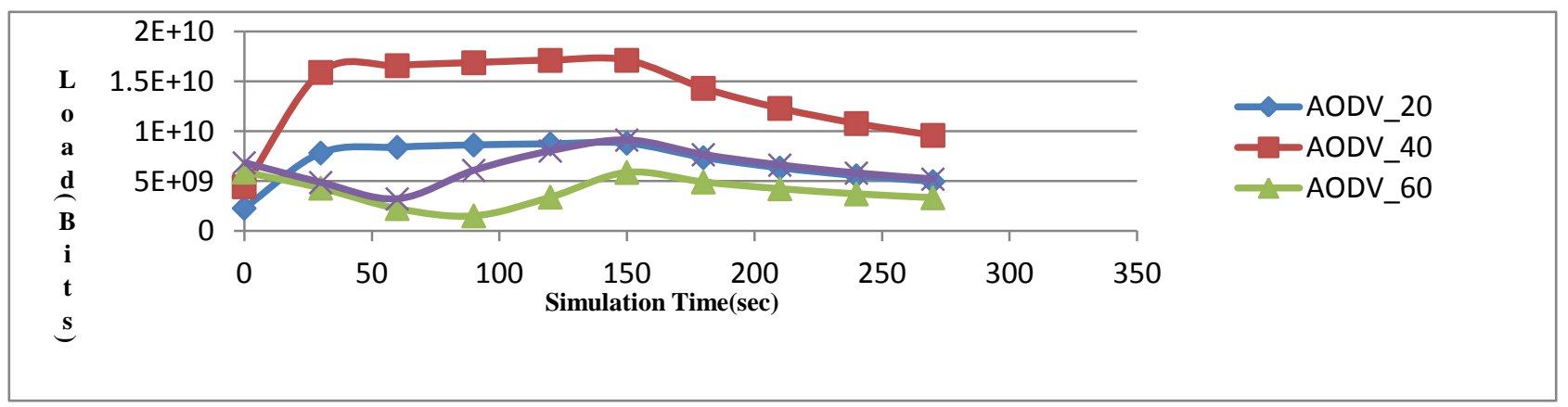

Figure 5: load of AODV

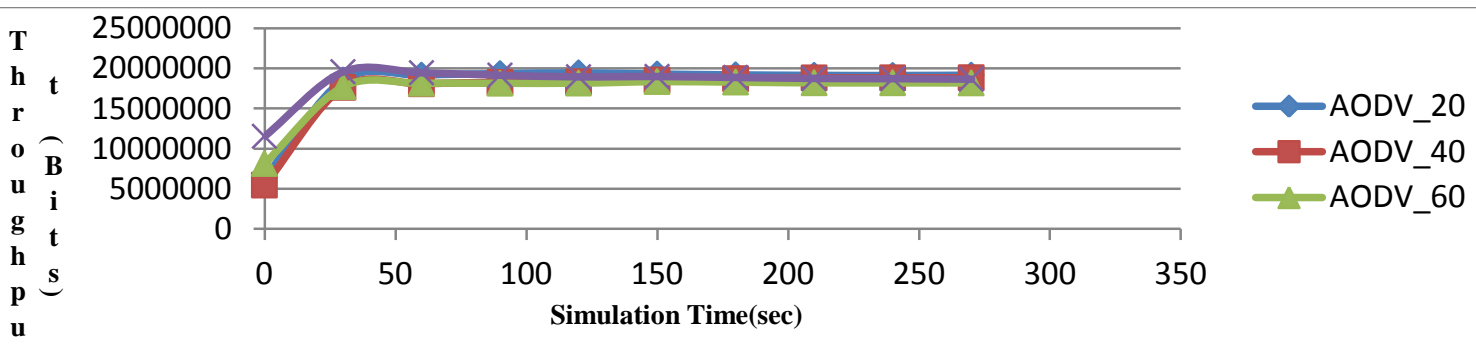

Figure 6: Throughput of AODV 
Results of OLSR: The Analysis of OLSR routing protocol have done with varying $(20,40,60,80)$ number of nodes. OLSR protocol was simulated in all the four scenarios by using all the two parameters such as: Load, Throughput. These two parameters can be checked in all the 20, 40, 60, 80 mobile nodes.

Load: In fig. 7 shows that network scenario with 20 mobile nodes is showing minimum load during simulation than network scenario with 40,60 and 80 mobile nodes. The network scenarios having 40,60 and 80 mobile nodes shows maximum load at the time of simulation.

Throughput: In fig. 8 shows that Network density with 20 mobile nodes shows maximum throughput than 40,60 and 80 mobile nodes.

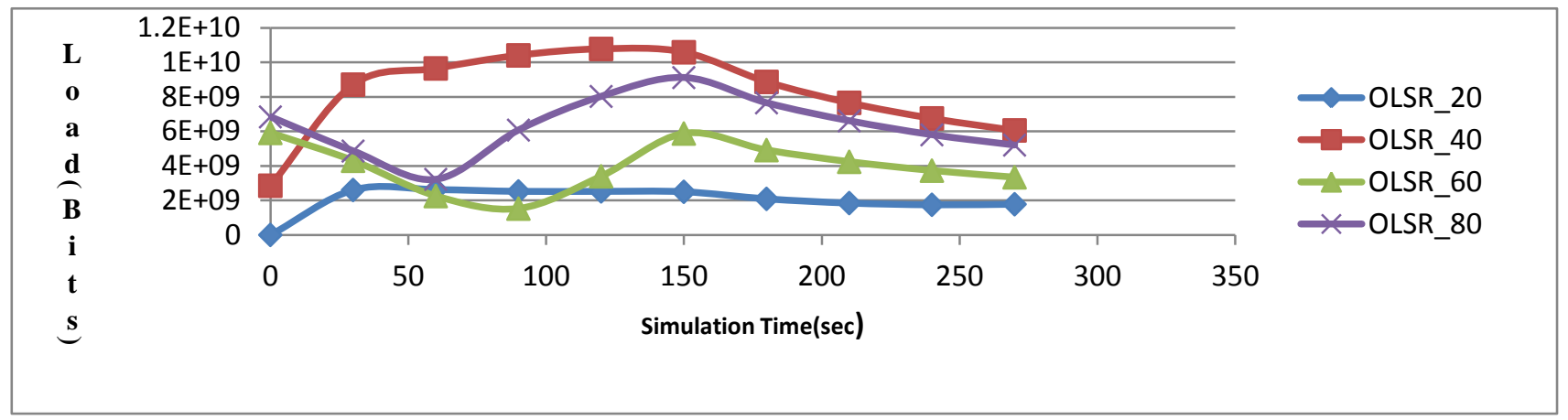

Figure 7: Load of OLSR

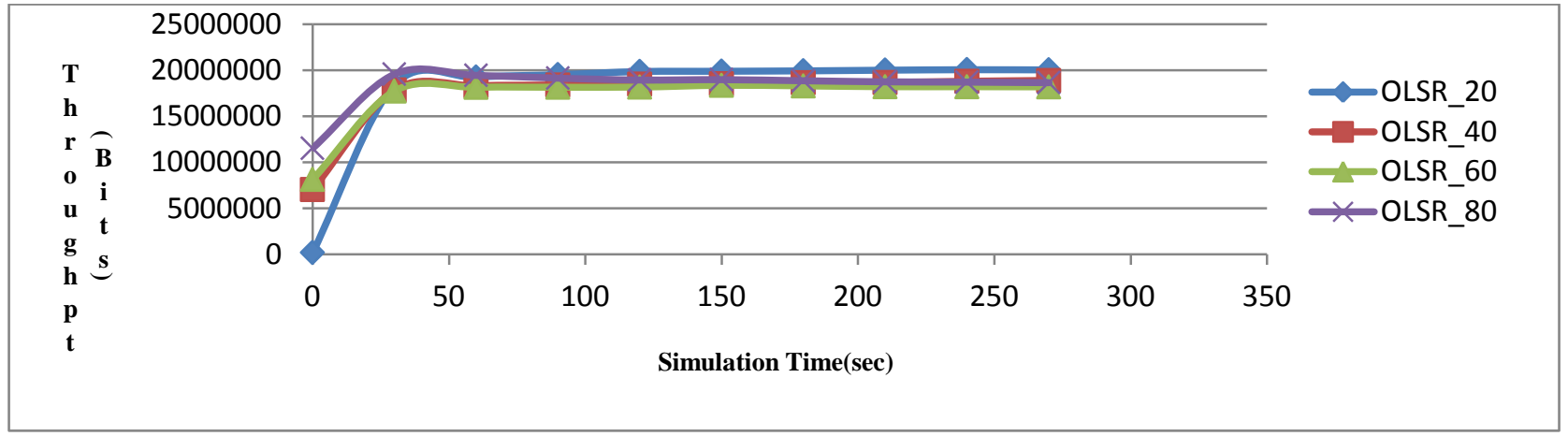

Figure 8: Throughput of OLSR

Results of GRP: The Analysis of GRP routing protocol have done with varying $(20,40,60,80)$ number of nodes. GRP protocol was simulated in all the four scenarios by using all the two parameters such as: Load, Throughput. These four parameters can be checked in all the 20, 40, 60, 80 mobile nodes.

Load: In fig. 9 shows that network density with 40 mobile nodes shows maximum load. Load can be measure in bitslsec. With increase in number of mobile nodes load increases network density with 20 mobile nodes shows minimum load.

Throughput: From the given results in fig. 10 shows that network density with 80 mobile nodes shows maximum throughput than 60, 40, 20 mobile nodes. With increase of mobile nodes in a network throughput also increases in case of GRP routing protocols. Because of the hybrid nature, protocol decides it's working according to the zones. For the nodes present in the range of a specific zone, the hybrid protocol behaves like a reactive protocol and for the nodes present out of the range, protocol behaves like a proactive protocol. With every increment of the node density, it has seen that there is a steep increment in the throughput of the networks.

\section{CONCLUSION}

A simulation based analysis of one reactive protocol (AODV), one proactive protocol (OLSR) and one hybrid routing protocol (GRP) has done under varying node densities by using different types of applications, such that High Definition Video Conferencing and High Load FTP. After running intensive simulations, it has been concluded that as the number of nodes increases in the network, throughput of the network configured by using a hybrid protocol, GRP increases proportionally, such that the throughput has increased by approximately $32 \%$ with each increment of nodes due to its boundary resolution feature in which, for a specific range protocol works like a reactive protocol and beyond the range, protocol begins to work like a proactive protocol. Though, increment has also seen in the throughputs of other two protocols, AODV and OLSR, but in comparison to the hybrid protocol, the increment in less. Among the reactive protocol (AODV) and proactive protocol (OLSR), the OLSR protocol outperforms the AODV protocol.

\section{FUTURE SCOPE}

There is always a scope of improving the results of work done by increasing the number of nodes and by configuring other present MANET routing protocols or by running the simulations for longer duration. Different performance evaluation metrics could be chosen to make the concluded results more justified. 


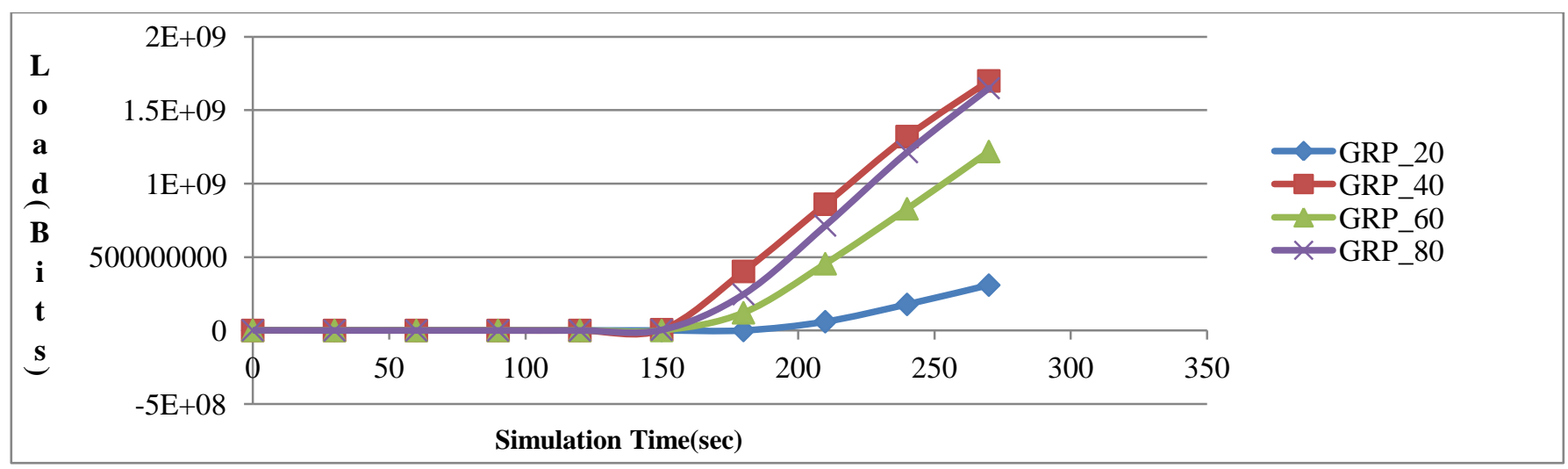

Figure 9: Load of GRP

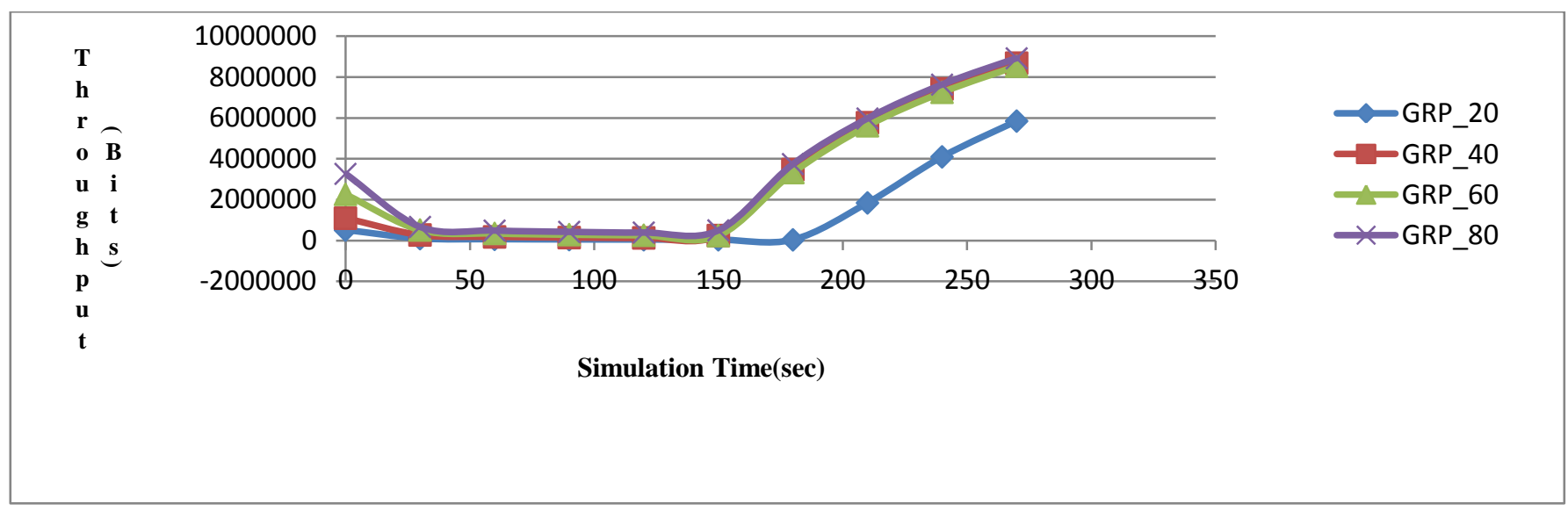

Figure 10: Throughput of GRP

\section{REFERENCE}

[1] Asad Amir Pirzada, Saad Khan, Marius Portmann, "Performance Comparison of Reactive Routing Protocols for Hybrid Wireless Mesh Networks." 2nd International Conference on Wireless Broadband and Ultra Wideband Communications (Aus Wireless 2007), p.p. 1-6, February 2007.

[2] Ashish Shrestha and Firat Tekiner, "On MANET Routing Protocols for Mobility and Scalability." International Conference on Parallel and Distributed Computing, Applications and Technologies, p.p. 451456, November 2009.

[3] Baldev Ram Mali and N.C. Barwar, "Performance of MANET Routing Protocols considering Impact of Node Density under Different Traffic Patterns." International Journal of Computer Applications (0975 - 8887) on Wireless Communication and Mobile Networks, vol-1, No.6, p.p. 26-30, January 2012.

[4] C.P. Agrawal, M.K Tiwari, O.P Vyas, "Evaluation of AODV protocol for varying mobility models of MANETs for ubiquitous computing." Third International Conference on Convergence and Hybrid Information Technology, vol.1, p.p. 769-774, November 2009.

[5] Dr.Aditya Goel, Ajaii Sharma, "Performance Analysis of Mobile Ad-hoc Network Using AODV Protocol."
International Journal of Computer Science and Security (IJCSS), p.p. 334-343, vol. 3, issue 5, December 2011.

[6] Ejiro .E. Igbesoko, Thaddeus Onyinye Eze, Mona Ghassemian "Performance Analysis of MANET Routing Protocols over Different Mobility Models." London Communication Symposium, p.p. 1-4, September 2010, London United Kingdom.

[7] Gunjesh Kant Singh, Harminder Singh Bindra, A. L. Sangal "Performance Analysis of DSR, AODV Routing Protocols based on Wormhole Attack in Mobile Ad-hoc Network." International Journal of Computer Applications, vol.26, No-5, p.p.38-41, July 2011.

[8] Hari Shankar Mewara, Saurabh Porwal "Throughput Performance Comparison of Reactive and Proactive Routing Protocols in Mobile Ad Hoc Networks using OPNET v14.5", IJCA Proceedings on Recent Trends in Information Technology and Computer Science, p.p. 914, February 2013.

[9] Juan Carlos Cano, Pietro Manzoni “A Performance Comparison of Energy Consumption for Mobile Ad Hoc Networks Routing Protocols." 8th International Symposium on Modeling, Analysis and Simulation of Computer and Telecommunication Systems (MASCOTS '00) , p.p. 1-8, September 2001, San Francisco California. 
[10] Kuldeep Vats, Mandeep Dalal, Deepak Rohila, Vikas Loura, "OPNET Based Simulation and Performance Analysis of GRP Routing Protocol". International Journal of Advanced Research in Computer Science and Software Engineering, vol.2, p.p. 119-122, March 2012

[11] Li Zhiyuan, "Geographic Routing Protocol and Simulation" 2009 Second International Workshop on Computer Science and Engineering, p.p 404-407, Guilin College of Aerospace Technology Guilin, Guangxi Province, China.

[12] M.K. Jeya Kumar and R.S. Rajesh, "Performance Analysis of MANET Routing Protocols in Different Mobility Models." IJCSNS International Journal of Computer Science and Network Security, vol.9, p.p. 2229, February 2009.

[13] Monika Rajput, pallavi khatri, alankar shastri, keshav solanki, "Comparison of Ad-hoc Reactive Routing Protocols using OPNET Modeler," International Conference on Computer Information Systems and Industrial Management Applications (CISIM), p.p. 530534, 2010.

[14] N. Adam, M.Y. Ismail et al., "Effect of Node density on Performances of Three MANET Routing Protocols." International Conference on Electronic Devices, Systems and Applications (ICEDSA2010), p.p. 321-325, October 2010.

[15] Naveen Bilandi, Harsh K Verma "Comparative Analysis of Reactive, Proactive and Hybrid Routing Protocols in MANET." International Journal of Electronics and Computer Science Engineering. vol. 1, No. 3, p.p. 16601667,2012

[16] N Vetrivelan and A V Reddy, "Performance Analysis of Three Routing Protocols for Varying MANET Size." In Proceedings of the International Multi Conference of Engineers and Computer Scientists (IMECS 2008), vol.2, p.p. 1178-1182, March 2008.

[17] Prof. Dr. C. A. Dhote, Prof M.A.Pund, Prof. R.S. Mangrulkar, "Hybrid Routing Protocol with Broadcast Reply for Mobile Ad hoc Network." International Journal of Computer Applications, p.p. 108-113, vol. 10, February 2010.

[18] Puneet kumar, bharadwaj, Shipra Sharma, Vandana Dubey, "Comparative Analysis of Reactive and Proactive Protocol of Mobile Ad-Hoc Network." International Journal on Computer Science and Engineering (IJCSE). vol.4, p.p 1281-1288, July 2012.

[19] Qingting Wei and Hong Zou, "Efficiency Evaluation and Comparison of Routing Protocols in MANETs." International Symposium on Information Science and Engineering 2008, vol.2, p.p. 175-177, 2008.

[20] Ricardo de Oliveira Schmidt, Marco Antônio Sandini Trentin, "MANETs Routing Protocols Evaluation in a
Scenario with High Mobility." IEEE Network Operations and Management Symposium (NOMS 2008), p.p. 883886, August 2008.

[21] R. Mohan, C.Rajan, Dr. N.Shanthi, “A Stable Mobility Model Evaluation Strategy for MANET Routing Protocols." International Journal of Advanced Research in Computer Science and Software Engineering. vol. 2, p.p.58-65, December 2012.

[22] Rajeswari.M, Maheswari.P.U, Bhuvaneshwari.S, Gowri.S, "Performance analysis of AODV, DSR, TORA and OLSR to achieve group communication in MANET." Fourth International Conference on Advanced Computing, ICoAC 2012 MIT, p.p.1-8. Anna university, Chennai, December 13-15, 2012.

[23] Sabina Barakovic and Jasmina Barakovic, "Comparative Performance Evaluation of Mobile Ad Hoc Routing Protocols." MIPRO, p.p. 518-523, May, 2010.

[24] Shaily Mittal and Prabhjot Kaur, "Performance Comparison of AODV, DSR and ZRP Routing Protocols in MANETs." International Conference on Advances in Computing, Control, and Telecommunication Technologies, p.p. 165-168, November 2009. IEEE Computer Society.

[25] Sukhchandan Lally and Ljiljana Trajković "Performance Analysis of Routing Protocols for Wireless Ad-Hoc Networks." Simon Fraser University Vancouver, p.p. 18, British Columbia, Canada, 2011.

[26] Shivendu Dubey and Rajesh Shrivastava, "Energy Consumption using Traffic Models for MANET Routing Protocols." International Journal of Smart Sensors and Ad Hoc Networks (IJSSAN), vol.1, p.p.84-89, 2011.

[27] Valentina Timcenko, Mirjana Stojanovic et al., "MANET Routing Protocols vs. Mobility Models: Performance Analysis and Comparison." 9th WSEAS International Conference on APPLIED INFORMATICS AND COMMUNICATIONS (AIC '09), p.p. 271-276, 2009

[28] Vasudha Arora and C. Rama Krishna, "Performance Evaluation of Routing Protocols for MANETs under Different Traffic Conditions." 2nd International Conference on Computer Engineering and Technology, vol. 6, p.p. 79-84, July 2010.

[29] Vikas singla and parveen kakkar "Traffic Pattern based performance comparison of Reactive and Proactive protocols of Mobile Ad-hoc Networks." International Journal of Computer Applications, vol. 5, p.p.16-20, August 2010.

[30] Xinjie Chang, "NETWORK SIMULATIONS WITH OPNET." Winter Simulation Conference, p.p. 307-314, 1999, Network Technology Research Center (School of EEE), Nanyang Technological University, Singapore 